\title{
Nanostructuring in Indirect Band-gap Semiconductor Using IR Femtosecond Double Pulses
}

\author{
Yasuhiko Shimotsuma $^{* 1}$, Tomoaki Sei ${ }^{1}$, Masaaki Sakakura ${ }^{2}$, Kiyotaka Miura ${ }^{1}$, Haruhiko Udono ${ }^{3}$ \\ ${ }^{* 1}$ Department of Material Chemistry, Graduate School of Engineering, Kyoto University, Kyoto \\ 615-8510, Japan \\ yshimo@func.mc.kyoto-u.ac.jp \\ ${ }^{2}$ Society-Academia Collaboration for Innovation, Kyoto University, Kyoto 615-8510, Japan \\ ${ }^{3}$ College of Engineering, Ibaraki University, Hitachi, Ibaraki 316-8511, Japan
}

\begin{abstract}
Periodic nanostructures inside an indirect band-gap semiconductor crystal have been induced by a focused beam of a femtosecond double-pulse. Such self-organized nanostructures in silicon are composed of strained silicon, which exhibit a high electric conductivity. On the other hand, the nanostructures have not been empirically formed in a direct band-gap semiconductor. We classify the observed phenomenon into two types based on the bandgap structure and propose the mechanism for nanostructuring. Since the scattering of phonons by the nanostructure in semiconductor leads to a low thermal conductivity, we have also proposed the possible application to the thermoelectric conversion devices.
\end{abstract}

DOI: $10.2961 /$ jlmn.2016.01.0007

Keywords: band gap, semiconductor, femtosecond laser, infrared, nanostructure

\section{Introduction}

It is well known that there are three ways to classify the semiconductor materials. The typical way is due to the material. The representative single-element semiconductors are silicon $(\mathrm{Si})$, germanium $(\mathrm{Ge})$ and diamond $(\mathrm{C})$. On the other hand, the gallium arsenide (GaAs) and gallium nitride $(\mathrm{GaN})$ is known as a typical compound semiconductor. Furthermore, based on the electrical classification, semiconductors are mainly classified into two categories of the intrinsic and the extrinsic. Another is an optical (or energy) classification. According to the energy band diagram, the semiconductors can be classified into the two types of the direct and the indirect band gap. It's also well known that the direct band gap semiconductors are exclusively used for optoelectronic devices such as laser diodes. Although micro- and nano-fabrication of wide bandgap semiconductor [1] including laser lift-off delamination technique [2] has been investigated by many researchers, few researches of the laser-direct-writing inside the semiconductor were reported [3]. Besides, the interaction between a semiconductor and ultrashort laser pulse is intensively studied in the various fields ranging from coherent phonon excitation [4], cavity-exciton polariton [5], to semiconductor-to-metal transition [6]. Especially nano- and micro-modified silicon surface can be successfully formed by irradiation with the ultrashort laser pulses [7]. Since the discovery of the laserinduced periodic surface structure (LIPSS) by Birnbaum nearly a half-century ago [8], many interesting researches have been carried out from the experimental [9] and theoretical [10] perspectives. Even now the formation mechanisms of LIPPS are under debate [11].

Furthermore, a decade ago, a new type of self-organized periodic nanostructure was observed inside transparent isotropic material such as $\mathrm{SiO}_{2}$ glass after an ultrafast laser pulse irradiation [12]. Under certain conditions, highly or- dered subwavelength structures with features smaller than $20 \mathrm{~nm}$, showing optical anisotropy, could be aligned perpendicularly to the laser polarization [13]. As opposed to LIPPS on the various materials (metal, semiconductor, dielectrics), nanogratings inside of the material were found only for handful materials $[14,15]$. The formation mechanism of nanogratings is also still a mystery $[12,16]$. More recently, we have also presented that the polarizationdependent periodic nanostructures were induced inside single-crystalline silicon (c-Si) by the infrared femtosecond double-pulses irradiation [17].

Here we report on the empirical classification of the nanostructuring inside various semiconductor materials of $\mathrm{GaN}, \mathrm{GaAs}$ and Si induced by the irradiation of IR femtosecond laser pulses, based on the energy band diagram. $\mathrm{GaN}$ of a wide bandgap semiconductor is one of key material for blue LED and power devices. Furthermore, GaN is expected as a material with various optical response attributed to its stable exciton $(\sim 28 \mathrm{meV})$. In the case of $\mathrm{GaN}$, polarization-dependent periodic nanostructures have not been observed in our experiments so far. This result was similar to the case of gallium arsenide (GaAs) and zinc oxide $(\mathrm{ZnO})$. On the other hand, we also present that the polarization-dependent periodic nanostructures were induced inside single-crystalline silicon (c-Si) by the infrared femtosecond double-pulses irradiation [17]. Such nanostructures consist of the strained silicon regions with a width of about $100 \mathrm{~nm}$ and align parallel to the laser polarization with a period of $200 \mathrm{~nm}$. The formation mechanisms would be interpreted in terms of the electrostrictive force through the interaction between electron-hole plasma and phonon. We have also confirmed that such nanostructures were also formed inside gallium phosphide (GaP) by the femtosecond laser irradiation. These results experimentally indicate that the self-organization inside materials 
triggered by ultrashort pulses irradiation is possibly associated with a direct or an indirect band gap.

\section{Femtosecond direct writing in GaN}

In the experiments we used a commercially available freestanding $\mathrm{GaN}$ substrate. In the experiments, a modelocked, regeneratively amplified Ti: $\mathrm{Al}_{2} \mathrm{O}_{3}$ laser system (Coherent; Legend), operating at $800 \mathrm{~nm}$ with $100 \mathrm{fs}$ pulse duration and $1 \mathrm{kHz}$ repetition rate was used. The laser beam was focused via a microscope objective (Nikon; LU Plan Fluor, $100 \times 0.90$ N.A.) at a depth of about $300 \mu \mathrm{m}$ below the GaN crystal sample surface. To reveal the effect on the photoinduced structure derived from the hexagonal wurtzite type crystal structure (Fig. 1), the spatial configuration between laser propagation and polarization was set according to its crystal structure. The pulse energy was controlled by a variable neutral density filter $(1 \sim 350 \mu \mathrm{J})$. The linear polarization orientation of laser beam was rotated by a half-wave plate. The typical irradiation time was $0.5 \mathrm{~s}$ (500 pulses). Photoluminescence spectra excited by femtosecond light pulses under liquid helium temperature of about $10 \mathrm{~K}$ were also taken by an optical multichannel analyzer (Hamamatsu Photonics; PMA-11). During this measurement, the sample was put in micro-cryostat (Oxford Instruments; Microstat HiResII). After polishing the sample surface to the depth of the beam waist location, the surface was analyzed by a scanning electron microscope (JEOL; JSM-6705F).
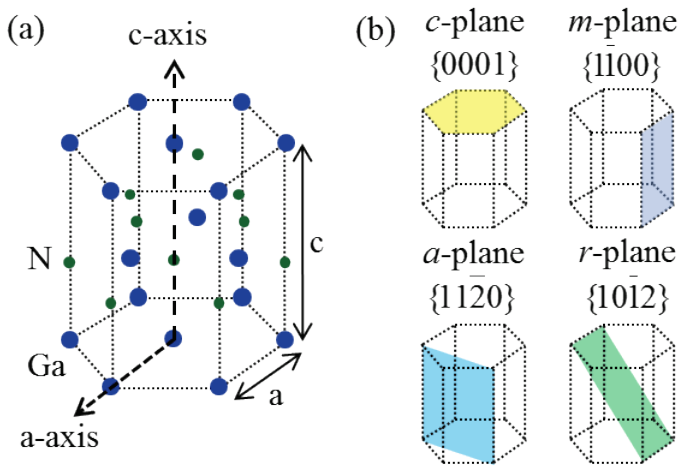

Fig. 1 (a) Crystal structure of GaN. (b) Typical crystal planes of c, m-, a- and r-plane.

(a)
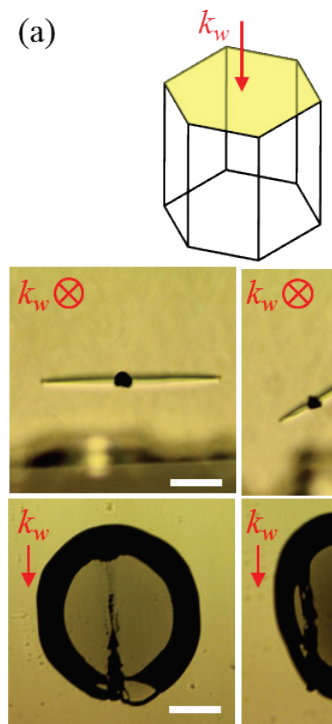

Fig. 2 Optical micrographs of the photoinduced structures inside $\mathrm{GaN}$ crystal by fs-laser irradiation in the direction (a) perpendicu- lar and (b) parallel to c-plane. Images in upper and lower rows were observed from c- and m-plane, respectively. $k_{\mathrm{w}}$ indicates the direction of laser propagation. Scale bars indicate $100 \mu \mathrm{m}$.

Fig. 2 shows typical optical micrographs of the photoinduced structures inside $\mathrm{GaN}$ crystal by laser irradiation in the direction perpendicular and parallel to c-plane. The induced structures were observed from two different view point normal to c- and $\mathrm{m}$-plane. These results apparently show that the black ring-shaped structures, which look like a crack viewing from the normal direction to c-plane, have been induced in the m- or a-plane. These structural changes in the normal direction to the c-plane can be also interpreted in terms of the elastic constants of wurtzite structure [18]. It was noted that such structure became large according to the number of laser shots. In order to reveal such structural changes, we performed the cross-sectional observation by using electron probe micro-analyzer (EPMA). Metallic gallium was detected in the black ring-shaped region (not shown here).

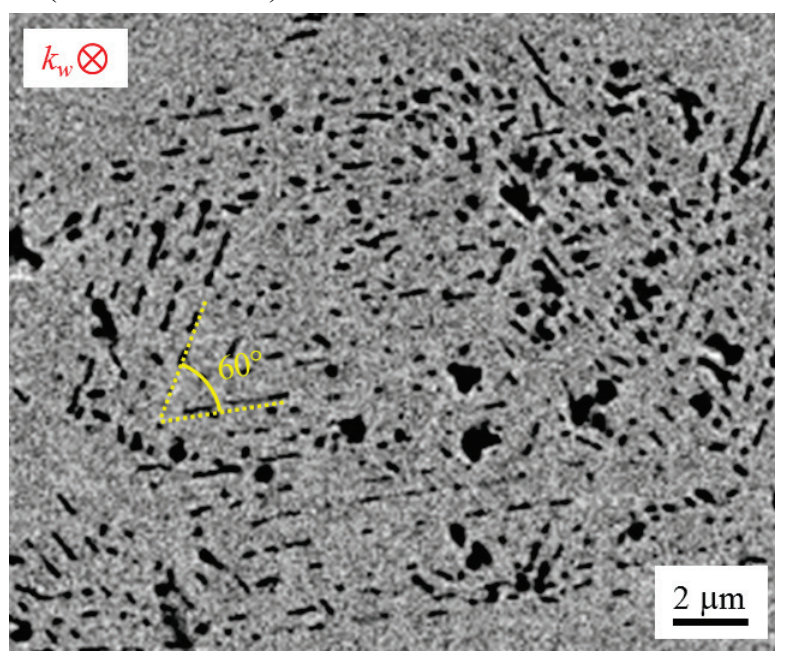

Fig. 3 Secondary electron image on the polished GaN surface in the center of the modified region. The laser irradiation direction is normal to the c-plane. Angle lines of $60^{\circ}$ are guides to the eye.

In addition, we have also carried out the SEM observation of the modified region inside $\mathrm{GaN}$ after the femtosecond laser irradiation (Fig. 3). No apparent polarizationdependent periodic nanostructures were observed at the center of the modified region. Note that the nanovoids were distributed in the triangular shape corresponding to the crystal structure (hexagonal wurtzite type) without depending on the laser polarization. The ring-shaped segregation of metallic gallium and the formation of nanovoids in the center of modified region were derived from the decomposition into $\mathrm{Ga}$ metal and $\mathrm{N}_{2}$ gas [19]. The chemical reaction taking place at the modified region can be expressed by:

$2 \mathrm{GaN}(s) \rightarrow 2 \mathrm{Ga}(l)+\mathrm{N}_{2}(g)$

We have confirmed that the laser damage probability also depends on the arrangement between the laser polarization and the crystal axis (Fig. 4). The difference in the laser damage probability between the laser polarization direction perpendicular to and parallel to c-axis was about 40 times or more. It should be noted that when the laser polarization direction is parallel to c-axis, the sample surface was also damaged by the laser pulses with energy of $280 \mu \mathrm{J}$ or more. To reveal the origin of the difference in 
this damage threshold, we observed photoluminescence (PL) during the laser irradiation at low temperature $(\sim 10$ $\mathrm{K})$.

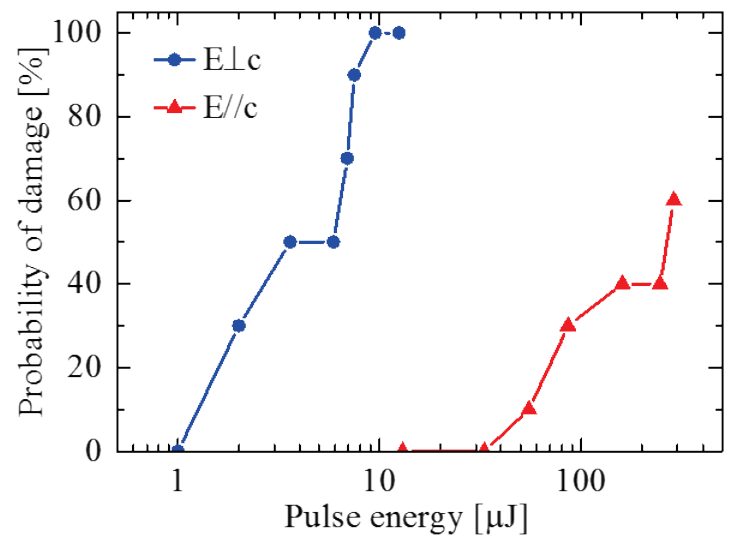

Fig. 4 Laser damage probability as a function of femtosecondlaser energy with the polarization direction parallel $(\mathrm{E} / \mathrm{c})$ or perpendicular $(\mathrm{E} \perp \mathrm{c})$ to $\mathrm{c}$-axis. The laser pulses were irradiated from the direction normal to $\mathrm{m}$-plane.
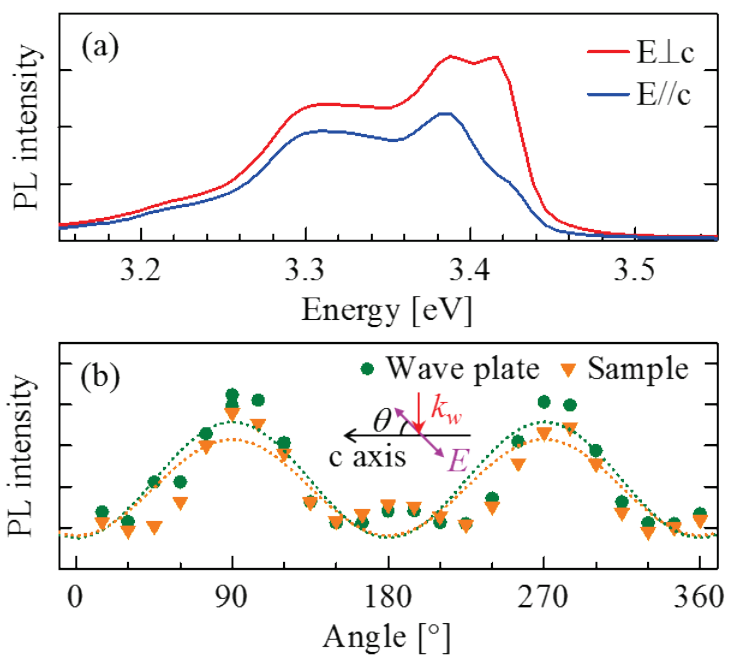

Fig. 5 (a) Photoluminescence spectra during the irradiation of the femtosecond light pulses with different polarization directions at $10 \mathrm{~K}$. (b) Intensity profiles of the photoluminescence from 3.2 to $3.4 \mathrm{eV}$ as a function of the angle between c-axis and polarization direction. The angle of $\mathrm{q}$ was changed by rotating the direction of waveplate or sample. Inset shows a schematic of the polarization and the propagation direction with respect to c-axis.

Fig. 5 shows PL spectra during the irradiation of the femtosecond light pulses with different polarization directions. Polarization-dependent PL intensity profiles as a function of the angle between c-axis and polarization direction were also shown. In view of the polarizationdependence of the measurement system, two different experiments of rotating sample or waveplate were carried out. In two distinct experiments, the PL intensity oscillated sinusoidally according to the angle between c-axis and the polarization direction. If the laser polarization direction is perpendicular to the c-axis, PL intensity reached maximum, resulting in the reduction of damage threshold. It is known that the valence band in wurtzite $\mathrm{GaN}$ is split into three subbands, A, B, and C, due to the crystal-field splitting and spin-orbit coupling [20]. The electronic transition relating free excitons involving a hole from such subbands depends on the light polarization [21]. Furthermore, we assumed the following mechanism. When the excitation light intensity is increased until the distance between the excitons reaches Bohr radius of an exciton, an exciton dissociates into an electron and a hole, and then an electron-hole plasma is formed. Finally the irradiated region are ionized via inverse Bremsstrahlung. As the result, the chemical reaction of (1) can occur at the photoexcited region.

\section{Femtosecond direct writing of nanostructure in $\mathrm{Si}$}

In the experiments we used commercially available undoped (100) silicon and gallium arsenide wafers. In order to overcome the bandgap limit, the experiments were performed using a mode-locked Cr: Forsterite femtosecond laser system equipped with a regenerative amplifier (Avesta; FREGAT-200), operating at $1.24 \mu \mathrm{m}$ with $110 \mathrm{fs}$ pulse duration and $1 \mathrm{kHz}$ repetition rate. To compensate a spherical aberration, the laser beam was focused inside silicon wafer through the objective lens with a correction collar (Olympus; LCPLN-IR, 100×, numerical aperture of 0.85 ). The induced structures inside silicon wafer were inspected by an optical microscope with infrared lamp (BHK; MAXIR) and InGaAs camera (Hamamatsu Photonics; C10633-13). After polishing the sample surface to the depth of the focal spot location, the polished sample surface was analyzed by a scanning electron microscope (JEOL; JSM-6705F).

At first we have confirmed that it is impossible to induce any structural changes inside c-Si by the femtosecond single-pulse-train even if the pulse energy is $600 \mu \mathrm{J}$ [22]. To promote the interaction between the excited electrons and the photon [23], we employed the femtosecond doublepulse configuration. The femtosecond double-pulses with the equally-divided pulse energy were employed in the experiments. The total pulse energy of the equally divided double-pulse with a delay time of 1 ps was typically set to $48 \mu \mathrm{J}$ (i.e., each pulse energy was $24 \pm 0.5 \mu \mathrm{J}$ ). Here two thresholds for the internal structural change $\left(E^{\text {in }}\right)$ and the surface ablation $\left(E^{\text {surf }}\right)$ should be considered. Furthermore, since the refractive index of $\mathrm{c}-\mathrm{Si}$ is as high as 3.5 at 1.24 $\mu \mathrm{m}$, the spherical aberration, leading to the decrease of laser energy density at the focus, should be also compensated in the case of the internal threshold.

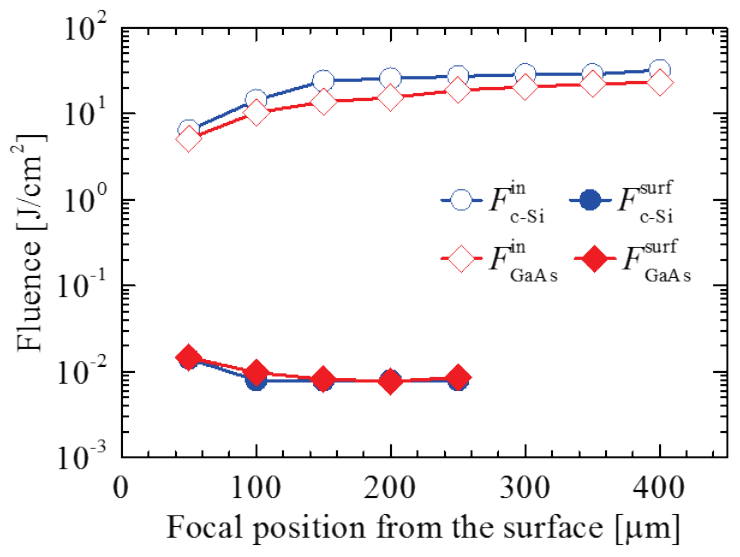

Fig. 6 Comparison of laser fluence thresholds for the internal structural change $\left(F^{\mathrm{in}}\right)$ and the surface ablation $\left(F^{\text {surf }}\right)$ as a function of the depth of focus from the sample surface $(d)$. 
Fig. 6 shows the fluence thresholds for the internal and the surface modification determined from the experiments as a function of the focus position from the sample surface (d). The laser fluence values at the focus and the surface were estimated by the following equations.

$$
\begin{aligned}
& F^{\text {in }}=\frac{E_{\mathrm{p}} T_{\mathrm{OL}}(1-R)}{\pi w_{0}{ }^{2}}, \quad w_{0}=0.61 \frac{n \lambda}{\mathrm{NA}} \\
& F^{\text {surf }}=\frac{E_{\mathrm{p}} T_{\mathrm{OL}}}{\pi r^{2}}, \quad r=\frac{d \mathrm{NA}}{\sqrt{1-\mathrm{NA}^{2}}}
\end{aligned}
$$

where $E_{\mathrm{p}}$ is the laser pulse energy, $T_{\mathrm{OL}}(=0.72)$ is the transmittance of objective, $R$ is the reflectance of material at the laser wavelength, $w_{0}$ is the laser beam waist at the focus, $r$ is the beam radius at the surface, and NA is the numerical aperture of objective. On the first inspection of the comparison by laser energy [17], the structural modification inside c-Si could be only observed in narrow laser energy window. By comparison of laser fluence thresholds for the internal structural change and the surface ablation, larger fluence value is required to structural modification in c-Si.
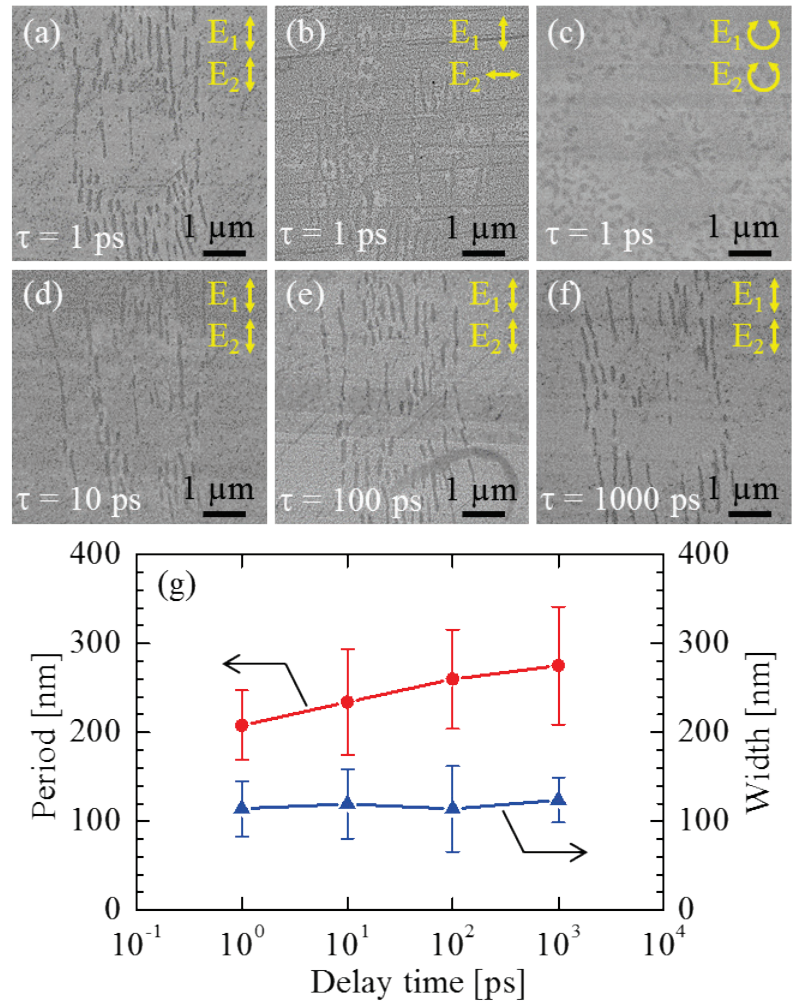

Fig. 7 Backscattering electron images (BEIs) on the polished sample surface to the depth of focal position of the femtosecond double-pulses with the vertical polarization directions (a), the orthogonal polarization directions (b) and the circular polarization directions (c). $E_{1}$ and $E_{2}$ indicate the polarization directions of the first and the second arriving pulse. The nanostructures induced by the vertically polarized femtosecond double-pulses with the delay time $(\tau)$ of 10 ps (d), 100 ps (e) and 1000 ps (f) were also shown. (g) Period and width of the photoinduced nanostructures in c-Si as a function of the delay time.

Fig. 7 (a) (c) show the backscattering electron images (BEIs) on the sample surface after polishing to the depth of focal position of the femtosecond double-pulses with the different polarization directions. Previously we have reported that the structural change of the modified region in silicon is analogous to the nanograting structure constituted by oxygen deficiencies in $\mathrm{SiO}_{2}$ glass [17]. In the case of $\mathrm{SiO}_{2}$ glass, the nanograting orientation is only defined by the last pulse train [13]. Erasure of the nanostructure requires sufficiently high temperatures as the induced oxygen defects are maintained up to the glass transition temperature $\left(\sim 1200^{\circ} \mathrm{C}\right)$. On the other hand, in the case of $\mathrm{c}-\mathrm{Si}$, the nanograting structures were aligned parallel to the polarization direction of the first arriving pulses [Fig. 7 (a), (b)]. More interestingly, in the case of the circular polarized double-pulses, the photoinduced nanostructure was doughnut shaped consisting of the dotted dark regions [Fig. 7 (c)]. It should be noted that both polarization-dependent periodic nanostructures inside c-Si and $\mathrm{SiO}_{2}$ glass are rewritable. In the case of $\mathrm{c}-\mathrm{Si}$, the stripe-like darker regions with a period of $\sim 200 \mathrm{~nm}$ in the backscattering electron images (BEIs), were also observed [17]. We have confirmed that such nanostructures in $\mathrm{c}-\mathrm{Si}$ were maintained after annealing at $1200{ }^{\circ} \mathrm{C}$ for $1 \mathrm{hr}$. Based on the fact that the c-Si is composed of the single composition, the tensile stress may be considered to be applied in such darker regions with a width of $\sim 100 \mathrm{~nm}$ in the BEIs corresponding to the lower-density material. In contrast, the brighter regions show the compressive stress. Ma et al. calculated the conduction band structure of Si under uniaxial [100] stress [24]. The results show that the electron mobility along the stress axis increases with tensile stress and decreases with compressive stress, while the opposite relation was obtained along the transverse directions to the stress axis. Furthermore, Li et al. have reported the strain effects on the thermal conductivity of silicon [25]. The results show that the thermal conductivity decreases continuously from compressive strain to tensile strain. According to these results, the darker regions in the BEIs can be assumed to indicate higher electrical and lower thermal conductivities. Indeed, such properties derived from the strained silicon was observed in the polarization-dependent nanostructure [17]. It should be noted that the periodic nanostructures inside c-Si were formed around the focal spot location, independent of the polarization directions. This is presumably because the electron plasma density generated by the first pulse in the central region is high enough to reflect the second pulse. Indeed, since the avalanche ionization is dominant process in the case of semiconductor [26], we assumed that the electron density excited in $\mathrm{c}-\mathrm{Si}$ is greater by several orders of magnitude than that of $\mathrm{SiO}_{2}$ glass after several picosecond time delay. Since it is known that the plasma density is strongly inhomogeneous both in the propagation direction and the radial direction [27], further investigation including delocalization of femtosecond radiation [28] should be conducted. We have also confirmed that the period of the induced nanostructure is increasing with increase of the delay time between the femtosecond double-pulses [Fig. 7 (d) (f)]. Although the period of the nanostructure ranging from $200 \mathrm{~nm}$ to $300 \mathrm{~nm}$ could be obtained by changing the delay time, the width was not substantially changed at about $100 \mathrm{~nm}$ [Fig. 7 (g)]. This is probably interpreted by the decrease of the plasma density of which interact with the second arriving pulse [12]. 
Although we have also carried out the same experiments for GaAs, no apparent periodic nanostructures were observed [17]. Furthermore, the polarization-dependent nanostructures could not be also observed in the case of GaN (Fig. 3). Another group has also observed that the formation of periodic strained layers associated with nanovoids inside a $\mathrm{SiC}$ single crystal [29]. Since a $\mathrm{SiC}$ is an indirect band gap semiconductor, their results are consistent with our interpretation that the nanostructures can be formed inside a material only if it is an indirect bandgap semiconductor. These phenomena could be interpreted in terms of the difference in the electron transition between indirect and direct bandgap semiconductor [17]. The longer relaxation time of the excited carrier in $\mathrm{c}-\mathrm{Si}$ promotes the interaction with the time-delayed second pulse, because the radiative recombination in indirect bandgap semiconductor requires a certain correlation of the positions of not only electrons and holes but also phonons. On the other hand, in the case of direct band gap semiconductor, the carrier (lifetime is sub-picosecond at room temperature) is relaxed before the arrival of time-delayed second pulse. As a result no apparent structural change could be observed. Although the high plasma density $\left(\sim 10^{21} \mathrm{~cm}^{-3}\right.$ at $800 \mathrm{~nm}$ for $\mathrm{SiO}_{2}$ ) can be generated during the beginning of the pulse, its lifetime is very short ( $\sim 150 \mathrm{fs})$ [27]. Therefore the following formation mechanisms of nanogratings in $\mathrm{SiO}_{2}$ are proposed: the non-radiative relaxation of excitons via relative long lifetime transition from self-trapped excitons (STE) to point-defects (their lifetime is several hundred picoseconds at room temperature) [30]. On the other hand, in the case of $\mathrm{c}-\mathrm{Si}$, the maximum carrier density excited by ultrashort laser pulses is limited to $\sim 10^{19} \mathrm{~cm}^{-3}$, simultaneously, it is unchanged through 100 ps timescale [31]. This long lifetime of excitons after the photoexcitation in silicon can be interpreted in terms of the phonon-assisted indirect transitions [32]. It should be pointed out that the delay time after the excitation by the first pulse is important factor for the nanostructure formation. In particular, a common feature of periodic nanostructure formation between c-Si and $\mathrm{SiO}_{2}$ glass is the existence of a transition with a long relaxation time. Although further investigations are required for understanding the mechanisms of self-organized nanostructure formation inside the silicon crystal, the formation of such periodic nanostructure is reminiscent of the mechanical vibration of the structure by the stimulated Brillouin scattering involving photonphonon coupling via electrostriction force [33].

\section{Conclusion}

We empirically classified the photoinduced nanostructures inside various semiconductor materials. In the case of the direct band gap semiconductor of $\mathrm{GaN}$ and GaAs, no apparent polarization-dependent periodic nanostructures were observed. In particular, the nanovoids were induced in the triangular shape corresponding to the GaN crystal structure. Furthermore, the photoinduced structure in GaAs could not be observed in our laser irradiation condition. On the other hand, polarization-dependent periodic nanostructures are observed inside single-crystal silicon after irradiation by a focused beam of a femtosecond double-pulse. Backscattering electron images of the irradiated spot reveal that a periodic structure of stripe-like dark regions with a width of $100 \mathrm{~nm}$ and a period of $200 \mathrm{~nm}$ are composed of the strained silicon. The period is controllable by changing the delay time between the femtosecond double-pulses. Since the strained silicon cause the decrease of effective mass of carrier, such nanostructures exhibit a high electric conductivity. Furthermore, the scattering of phonon by a periodic nanostructure leads a low thermal conductivity. Such self-organized nanostructures are aligned parallel to the polarization direction of the first arriving pulse in the femtosecond double-pulses. We have empirically found that this phenomenon can be induced only if it is indirect band gap semiconductor. Apart from the fundamental importance of the observed phenomenon, the induced nanostructures could be useful for the self-contained thermoelectric devices.

\section{Acknowledgments}

This work was partially supported by JSPS KAKENHI Grant Number 26630129, The Thermal \& Electric Energy Technology Foundation, Tokuyama Science Foundation, Cross-Ministerial Strategic Innovation Promotion (SIP) Program and Industry-Academia Collaborative R\&D Programs (Super Cluster Program).

\section{References}

[1] S. Nakashima, K. Sugioka and K. Midorikawa: Appl. Surf. Sci., 255, (2009) 9770.

[2] T. Ueda, M. Ishida and M. Yuri: Jpn. J. Appl. Phys. 50, (2011) 041001.

[3] E. Ohmura, F. Fukuyo, K. Fukumitsu and H. Morita: J. Achievement Materials Manufacturing Eng. 17, (2006) 381.

[4] M. Hase, M. Kitajima, A. M. Constantinescu and H. Petek: Nature 426, (2003) 51.

[5] H. M. Gibbs, G. Khitrova and S. W. Koch: Nature Photon. 5, (2011) 275.

[6] S. K. Sundaram and E. Mazur: Nature Mater. 1, (2002) 217.

[7] M. Y. Shen, C. H. Crouch, J. E. Carey and E. Mazur: Appl. Phys. Lett. 85, (2004) 5694.

[8] M. Birnbaum: J. Appl. Phys. 36, (1965) 3688.

[9] J. Bonse, J. Krüger, S. Höhm and A. Rosenfeld: J. Laser Appl. 24, (2012) 042006.

[10] J. E. Sipe, J. F. Young, J. S. Preston and H. M. van Driel: Phys. Rev. B 27, (1983) 1141.

[11]M. Hashida, Y. Ikuta, Y. Miyasaka, S. Tokita and S. Sakabe: Appl. Phys. Lett. 102, (2013) 174106.

[12] Y. Shimotsuma, P. G. Kazansky, J. Qiu and K. Hirao: Phys. Rev. Lett. 91, (2003) 247405.

[13] Y. Shimotsuma, M. Sakakura, P. G. Kazansky, M. Beresna, J. Qiu, K. Miura and K. Hirao: Adv. Mater. 22, (2010) 4039.

[14] Y. Shimotsuma, K. Hirao, J. Qiu and P. G. Kazansky: Mod. Phys. Lett. B 19, (2005) 225.

[15]S. Richter, C. Miese, S. Döring, F. Zimmermann, M. J. Withford, A. Tünnermann and S. Nolte: Opt. Mater. Express 3, (2013) 1161.

[16] V. R. Bhardwaj, E. Simova, P. P. Rajeev, C. Hnatovsky, R. S. Taylor, D. M. Rayner and P. B. Corkum: Phys. Rev. Lett. 96, (2006) 057404. 
[17]M. Mori, Y. Shimotsuma, T. Sei, M. Sakakura, K. Miura and H. Udono: Phys. Status Solidi A 212, (2015) 715.

[18]A. F. Wright: J. Appl. Phys. 82, (1997) 2833.

[19]T. Ueda, M. Ishida and M. Yuri: Jpn. J. Appl. Phys. 50, (2011) 041001.

[20]M. A. Reshchikov and H. Morkoç: J. Appl. Phys. 97, (2005) 061301

[21]P. P. Paskov, T. Paskova, P. O. Holtz and B. Monemar: Phys. Status Solidi B 228, (2001) 467.

[22] Y. Ishikawa, Y. Shimotsuma, A. Kaneta, M. Sakakura, M. Nishi, K. Miura, K. Hirao, Y. Kawakami, Proc. of SPIE 8243, (2012) 82430N.

[23]K. Sugioka, M. Iida, H. Takai and K. Micorikawa: Opt. Lett. 36, (2011) 2734.

[24] J. L. Ma, Z. F. Fu, Q. Wei and H. M. Zhang: Silicon 5, (2013) 219.

[25] X. Li, K. Maute, M. L. Dunn and R. Yang: Phys. Rev. B 81, (2010) 245318.
[26]P. P. Pronko, P. A. VanRompay, C. Horvath, F. Loesel, T. Juhasz, X. Liu and G. Mourou: Phys. Rev. B 58, (1998) 2387.

[27] M. Lancry, N. Groothoff, B. Poumellec, S. Guizard, N. Fedorov and J. Canning: Phys. Rev. B 84, (2011) 245103.

[28] V. V. Kononenko, V. V. Konov and E. M. Dianov: Opt. Lett. 37, (2012) 3369.

[29] T. Okada, T. Tomita, S. Matsuo, S. Hashimoto, Y. Ishida, S. Kiyama and T. Takahashi: J. Appl. Phys. 106, (2009) 054307.

[30]D. Wortmann, M. Ramme and J. Gottmann: Opt. Express 15, (2007) 10149.

[31] A. Mouskeftaras, A. V. Rode, R. Clady, M. Sentis, O. Utéza and D. Grojo: Appl. Phys. Lett. 105, (2014) 191103.

[32]T. Suzuki and R. Shimano: Phys. Rev. B 83, (2011) 085207.

[33]R. Boyd: Nonlinear Optics, Academic Press, third edition, New York 2009.

(Received: May 22, 2015, Accepted: January 14, 2016) 